B. Wüthrich I. C. Blötzer

\section{IgE-vermittelte Nahrungsmittelallergien Typ C: Der seltenere Typ einer Nahrungsmittelallergie?}

Eine Kasuistik von 16 Fällen

\author{
IgE-Mediated Food Allergy Type C: A Rare Type of Food Allergy? \\ Report on 16 cases
}

\section{Zusammenfassung}

Nahrungsmittelallergien Typ C werden als selten beschrieben. Sie kommen vor allem bei erwachsenen Frauen vor, welche auch häufig keine atopische Disposition aufweisen. Sie beruhen auf einer Sensibilisierung über den Gastrointestinaltrakt gegen wenige, relativ stabile Nahrungsmittelproteinen. Unter $87 \mathrm{~Pa}$ tienten mit gesicherter Nahrungsmittelallergie im Jahre 1998 konnten 16 Patienten (18,4\%), im Alter von 12 bis 58 Jahren, dem Typ C zugeordnet werden. Zwei Drittel der Patienten sind weiblich und nur in knapp ein Fünftel ließ sich eine persönliche Atopie nachweisen. Bei elf der 16 Fälle konnten als Allergene tierische Eiweiße (5-mal Krustentiere, 2-mal Fleisch, je 1-mal Fisch, Geflügel, Ei und Milch) ermittelt werden, bei fünf anderen waren Nahrungsmittelallergene pflanzlichen Ursprungs (je 1-mal Getreide, Paranuss, Kiwi, Feige, Karotte/Kartoffel und Curry) im Spiele. In vier Fällen war die Auslösung multifaktoriell. Bei einem Fall war der wahrscheinliche Sensibilisierungsweg perkutan, im Sinne eines Kontakturtikariasyndroms.

\section{Abstract}

Food allergy Type $\mathrm{C}$ is described as rare. The majority of affected persons are females, who get sensitized via the gastrointestinal tract to selected, stable food proteins. Among 87 persons with proven IgE-mediated food allergy seen at the Allergy Unit of the University Hospital of Zürich during the year 199816 patients (18.4\%), aged between 12 and 58 years, belonged to the type C. $62.5 \%$ were females. Only $18.8 \%$ had a personal history of atopic diseases. Food of animal origin (crustaceans [ $n=5]$, meat $[n=2]$, fish, poultry, egg and cow's milk [1 time each]), were predominant as food allergens. Plant food allergens as Brazilnut, kiwi, fig, wheat, carrot/potatoes and curry elicited symptoms in five other patients after ingestion. In one previoulsy nonatopic patient the way of sensitization was the skin due to occupational contact with fish and salad (contact urticaria syndrome). This may represent a fourth type $\mathrm{D}$ of food allergy.

\section{Einleitung}

Nahrungsmittelallergien wurden bisher - gemäß Positionspapier der Europäischen Akademie für Allergologie und klinische Immunologie - nach pathogenetischen Gesichtspunkten eingeteilt $[1,2]$. Entsprechend der Art der Immunreaktionen kann man IgE-vermittelte, immunkomplex-bedingte, zytotoxische und T-Zell-vermittelte Reaktionen beobachten [3]. Pichler hat 1998 [4] eine Klassifikation der IgE-vermittelten Nahrungsmittelallergien in drei Typen A, B und C vorgeschlagen, welche die vom Alter abhängige unterschiedliche Häufigkeit und Verschiedenartigkeit des Allergenspektrums sowie die unterschiedliche Stabilität von Nahrungsmittelallergenen erklärt. Sie beruht auf dem grundsätzlich unterschiedlichen Sensibilisierungsweg der Nahrungsmittelallergien bei Kindern und Erwachsenen. Sie berücksichtigt den natürlichen Verlauf, die Toleranzentwicklung und die starke Altersabhängigkeit der Nahrungsmittelallergien sowie die molekulare Charakterisierung wichtiger Allergene und deren Kreuzreaktionen. Das Ziel dieser Klassifikation ist, zu 
einem besseren Verständnis von Nahrungsmittelallergien beizutragen.

Klassifikation von IgE-vermittelten Nahrungsmittelallergien

Die drei Typen A, B und C sind nach Pichler [4] folgendermaßen charakterisiert:

- Nahrungsmittelallergien Typ A

Diese Art von Nahrungsmittelallergie tritt im Säuglings- oder Kleinkindesalter auf. Dabei erfolgt die Sensibilisierung über den Gastrointestinaltrakt mit relativ stabilen Allergenen. Proteine aus Milch, Ei, Fisch und Erdnuss stellen die wichtigsten Allergene dar [5]. Mit Reifung der oralen Toleranz bildet sich die Allergie zurück oder Beschwerden können häufig ganz verschwinden. Diese Toleranz ist allergenabhängig. Sie wird häufig bei Milch-, Eier-, Soja- und Weizenallergie beobachtet, tritt jedoch nur selten bei Fisch- und Erdnussallergie ein.

- Nahrungsmittelallergie Typ B

Ältere Kinder, Jugendliche und Erwachsene sind von Nahrungsmittelallergien Typ B betroffen. Die Sensibilisierung erfolgt über den Respirationstrakt und nicht über den Gastrointestinaltrakt, da in diesem Alter die orale Toleranz bereits stabiler ist als die respiratorische Toleranz. Eine aerogene Sensibilisierung kann - unabhängig davon, ob sie klinisch manifest oder subklinisch verlaufend ist - die orale Toleranz umgehen und so zu Nahrungsmittelallergien führen. Dabei lösen aerogene Allergene (Pollen, Latex) die Bildung von IgE aus, welche mit ähnlichen Proteinen in Nahrungsmittel (Obst, Gemüse, Nüsse) kreuzreagieren [6-12]. Art und Lokalität der klinischen Symptomatik werden durch die Stabilität der kreuzreagierenden Nahrungsmittelallergene bestimmt, wobei die meisten Patienten nur milde Beschwerden, z. B. nur ein orales Allergiesyndrom (OAS) [13] aufweisen. In Mitteleuropa ist dies die häufigste Form von Nahrungsmittelallergien [13 - 16].

- Nahrungsmittelallergie Typ C

Patienten mit Nahrungsmittelallergie vom Typ C litten weder in ihrer Kindheit an Nahrungsmittelallergien noch sind sie später respiratorisch sensibilisiert worden. Es handelt sich meistens um erwachsene Frauen, welche - im Gegensatz zu Typ A und B - häufig keine atopische Disposition aufweisen [17]. Die Sensibilisierung erfolgt im Gastrointestinaltrakt gegen wenige, relativ verdauungsstabile Proteine. Nahrungsmittelallergien vom Typ C werden als selten angegeben.

Bei der Auswertung von 87 konsekutiven Fällen mit gesicherter Nahrungsmittelallergie der Allergiestation der Dermatologischen Universitätsklinik Zürich aus dem Jahr 1998, welche im Rahmen einer medizinischen Dissertation ausgewertet wurden [18], konnten 7 Patienten (8\%) dem Typ A, 64 (73,6\%) dem Typ B und $16(18,4 \%)$ dem Typ C zugeordnet werden. Im diesem Jahr wurden insgesamt 5521 Patienten abgeklärt, was einem prozentualen Anteil von Nahrungsmittelallergien (alle drei Typen inklusive) von lediglich 1,6\% entspricht. Diese Zahlen verdeutlichen einmal mehr die Diskrepanz, die besteht zwischen subjektiv empfundenem Vorhandensein einer Nahrungsmittelallergie und der tatsächlichen Häufigkeit in der Bevölkerung oder gar in einer spezialisierten Allergiepoliklinik. Wegen der geringen Anzahl an Patienten vom Typ A - kindliche Allergien werden in der Regel beim Pädiater oder im Kinderspital abgeklärt - ist die Ver- teilung der drei Typen nicht repräsentativ. Diese Zahlen bestätigen jedoch die allgemeinen Kenntnisse, dass unter Jugendlichen und Erwachsenen Nahrungsmittelallergien Typ B, aufgrund von Kreuzreaktionen zwischen aerogenen Allergenen und Nahrungsmitteln vorherrschen.

\section{Patienten und Methoden}

Im Folgenden werden die Krankengeschichten aller Patienten mit Nahrungsmittelallergie Typ C kurz beschrieben und die Testergebnisse tabellarisch dargestellt (Tab.1). Die Diagnose einer Nahrungsmittelallergie erfolgte im Rahmen der Routine-Arbeit auf der Allergiestation nach dem bewährten Verfahren von Anamnese, Hauttestung mit sämtlichen Modifikationen (Prick, Prick-Prick, Intrakutan, Scratch), IgE-Bestimmungen (RAST/CAP) und - falls notwendig oder im Rahmen von Studien - mit doppelt-blinden, plazebo-kontrollierten oralen Provokationstesten mit Nahrungsmitteln (DBPCFC) [19]. Die Inhalationsallergene wurde von der Firma ALK, Hörsholm, DK, die Nahrungsmittelallergen-Extrakte von der Firma Stallergènes Lab., Fresnès, $\mathrm{F}$ und die Gewürze von der Firma Allergopharma, Reinbek b. Hamburg, D, bezogen. Die spezifische IgE-Bestimmung erfolgte im allergologischen Laboratorium der Allergiestation Zürich nach den Angaben des Herstellers (Pharmacia Diagnostik, Uppsala, S).

Diese Kasuistik veranschaulicht, dass unter Jugendlichen und Erwachsenen durchweg Nahrungsmittelallergien auftreten können, auch wenn der Betroffene nicht unter manifesten Atopien wie Heuschnupfen, Asthma oder Neurodermitis, leidet.

\section{Kasuistik}

\section{Fall 1}

Im Alter von 51 Jahren entwickelte die Patientin ohne atopische Vorerkrankungen erstmals eine generalisierte Urtikaria kombiniert mit Quincke-Ödem und Flush nach dem Verzehr von Meeresfrüchten in Teneriffa. Eine Woche später trat nach Verspeisen eines Salattellers im gleichen Restaurant erneut eine generalisierte Urtikaria mit Benommenheit und Dyspnoe auf. Die später auf der Allergiestation durchgeführte Abklärung war bezüglich Atopie-Screening (Prick-Teste mit Inhalationsallergenen, inkl. Hausstaubmilben und Kuhmilch) negativ. Sowohl hauttestmäßig als auch serologisch (RAST/CAP) ergaben sich positive Sensibilisierungen gegenüber Krevetten (Garnelen) (Abb.1), Krabben und Hummer. In Bezug auf die zweite Episode ließen sich keine relevanten Sensibilisierungen auf Salate/Gemüse nachweisen; die von der Patientin verdächtigten Tomaten und Karotten wurden im oralen Provokationstest problemlos toleriert. Es ergab sich anamnestisch die Wahrscheinlichkeit, dass die Symptomatik durch einen mit Krevetten oder anderen Krustazeen kontaminierten Salat ausgelöst worden war. Diese Nahrungsmittelallergie ist eine Monosensibilisierung, welche bei negativem AtopieScreening und unabhängig von aerogenen Allergenen und Kreuzreaktionen im Sinne des Krustazeen-Hausstaubmilben-Syndroms [20-22] nach dem 50. Lebensjahr aufgetreten ist. Sie erfüllt alle von Pichler [4] genannten Kriterien einer Nahrungsmittelallergie Typ C und ist somit als ein typisches Beispiel einer solchen zu erachten. 
Tab. 1 Nahrungsmittelallergie vom Typ-C-Patienten Nr. 1-16 (Einzelheiten: siehe Text)

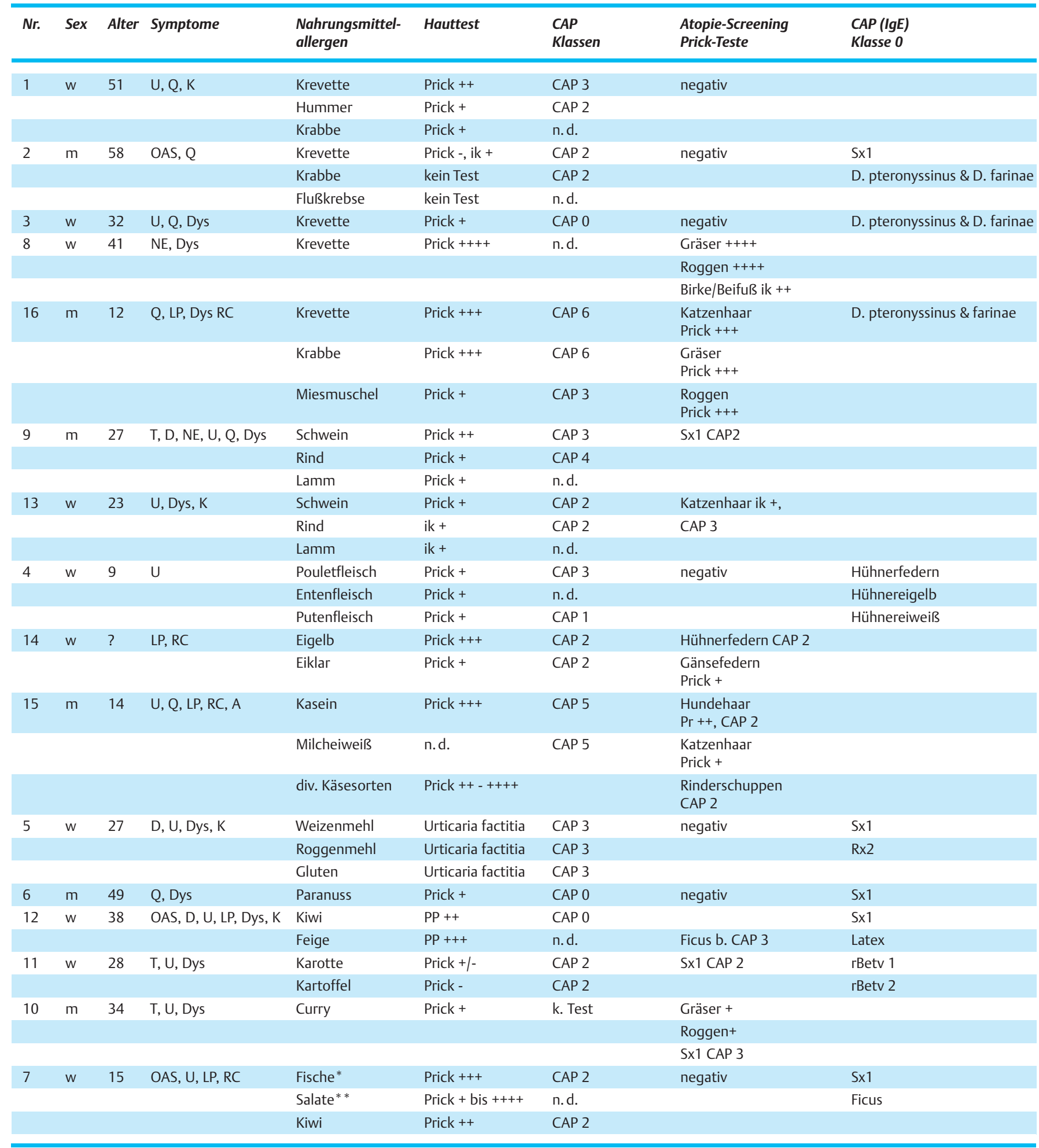

${ }^{*}$ diverse Fische (Prick): Süßwasserfische (+++), Dorsch (+++), Sardine $(+++)$, Thunfisch (+++), ${ }^{* *}$ diverse Salate (Prick): Rosso (++++), weißer Chicorée $(+++)$, Lollo $(+++)$, Kopfsalat (+), Kresse (+), Nüssli (Scratch+), Tomate $(+)$, Knoblauch $(+++)$

Legende: $\mathrm{A}=$ Asthma/Bronchospasmus, $\mathrm{D}=$ Diarrhö, Dys = Dyspnoe, ik = Intrakutantest, $\mathrm{K}=$ Kollaps, LP = Larynx-Pharynx-Ödem, OAS = Orales Allergiesyndrom, n. d. = nicht durchgeführt, $\mathrm{NE}=$ Nausea, Emesis, $\mathrm{Q}=$ Quincke-Ödem, RC = Rhinitis/Conjunctivitis, $\mathrm{T}=$ Tenesmen, Blähungen, $\mathrm{U}=\mathrm{Urtikaria}$ 


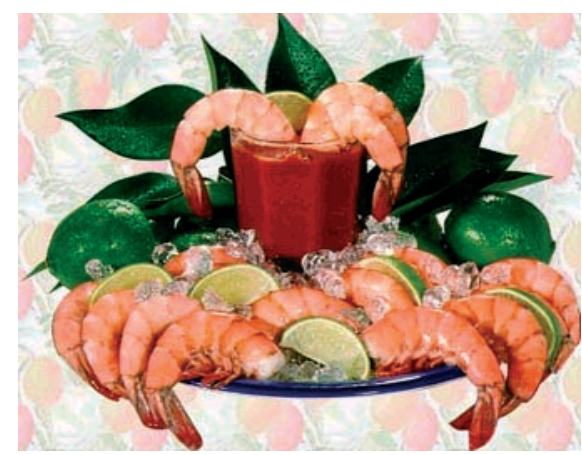

Abb. 1 Krevetten (Garnelen): das häufigste Allergen bei Nahrungsmittelallergien Typ C: Sensibilisierung und Auslösungauch bei Nichtatopikern und unabhängig von einem „HausstaubmilbenKrustazeen-Syndrom" möglich (vgl. Fälle Nr. 1-5).

\section{Fall 2}

Bei dem bis anhin atopisch nicht vorbelasteten 58-jährigen Patienten trat nach einer reichlichen Mahlzeit mit Süßwasserkrebsen kurz nach dem Essen ein generalisierter Pruritus im Mundbereich und ca. 3 bis 4 Stunden später ein Quincke-Ödem mit diffuser Gesichtsschwellung auf. Testmäßig konnten leichte Sensibilisierungen gegen Krevetten (Abb.1) und Krabben objektiviert werden (Prick-Test negativ; Intrakutan-Test +; sIgE CAP Klasse 2; spezifische IgE auf beide Hausstaubmilben negativ). Bei dieser eher schwachen Sensibilisierung spielt möglicherweise auch die Menge der eingenommenen Krustazeen eine Rolle. Zudem verfügen Krustazeen auch über histamin-liberierende Eigenschaften [21].

\section{Fall 3}

Während mehreren Monaten vor der allergologischen Abklärung erlitt die 32-jährige Patientin akut-rezidivierende Episoden mit Dyspnoe und Urtikaria nach dem Verzehr von Krevetten (Abb. 1). Bei negativen Prick-Testen auf Routine-Allergene war das auslösende Nahrungsmittel im Prick-Test deutlich positiv. Eine Neigung zu asthmatischen Beschwerden konnte bei unauffälliger Lungenfunktion und normalem Methacholin-Bronchoprovokationstest nicht objektiviert werden.

\section{Fall 4}

Innert fünf Minuten nach dem Verzehr von Krevetten in einem asiatischen Essen verspürte die 41-jährige Patientin retrosternale Schmerzen und Engegefühl mit Dyspnoe und erbrach daraufhin. Die Anamnese bezüglich Pollinose und andere Nahrungsmittelallergien im Sinne eines OAS war negativ. In den Hauttestungen war eine eindeutige Sensibilisierung gegen Krevetten ohne Sensibilisierung gegen Hausstaubmilben nachweisbar. Trotz positiven Prick-Tests auf verschiedene Pollen (Gräser-, Roggen-, Birke und Beifußpollen) im Atopie-Screening sowie geringfügig positiven Hauttests ohne klinische Relevanz bei einigen Nahrungsmitteln (Stangensellerie, Sellerie, Karotte, Kartoffel, Baumnuss und Mandeln) kann man in diesem Fall von einer Nahrungsmittelallergie vom Typ C bei monovalenter Sensibilisierung gegen Krevetten sprechen (Abb.1). Es besteht jedoch eine latente Birken- und Beifußpollenassoziierte Nahrungsmittelallergie, welche sich noch später als Nahrungsmittelallergie Typ B manifestieren könnte.

\section{Fall 5}

Seit dem 12. Lebensjahr traten beim 16-jährigen Patienten jeweils nach dem Verzehr von Krustazeen und Muscheln innerhalb von Minuten Lid- und Lippenschwellungen mit rhinokon- junktischen Beschwerden und Dyspnoe auf. Außerdem beklagte der Patient seit seiner Kindheit einen Heuschnupfen. Im Prickund CAP-Test (Klasse 6) ließ sich eine hochgradige Sensibilisierung auf Krevetten, Krabben (je CAP Klasse 6) und auf Miesmuschel (CAP Klasse 3) nachweisen. Im Atopie-Screening, außer Gräser- und Roggen-Pollen, waren noch Katzenhaare stark positiv, jedoch nicht Hausstaubmilben (D. farinae und D. pteronyssinus), auch nicht serologisch. Somit handelt es sich bei diesem Patienten um eine monovalente Sensibilisierung gegen Krustazeen und Mollusken bei einem Atopiker mit Pollinose, jedoch ohne Kreuzreaktion zu Hausstaubmilben (Abb.1).

\section{Fall 6}

Der Patient leidet seit seinem 27. Lebensjahr an rezidivierender, akuter, generalisierter Urtikaria, gelegentlich kombiniert mit Dyspnoe, Quincke-Ödem, Durchfall, Nausea und Emesis. Dem Patienten fiel auf, dass diese Episoden häufig innerhalb von Stunden nach dem Verzehr von Bratwürsten bzw. anderen Fleischwaren auftraten, wobei psychischer Stress, körperliche Anstrengung sowie gleichzeitiger Genuss von Rotwein die Symptomatik begünstigte. Entsprechend der Anamnese waren die Haut- und Serologie-Teste bezüglich verschiedener Fleischsorten (Schwein, Rind, Lamm, Pferd, Reh, Kaninchen) und Würste positiv (Abb. 2). Anamnestisch war keine persönliche Atopie eruierbar, das Atopie-Screening ist mit Ausnahme von Sx1 (CAP 2 = 3,22 kU/l) negativ.

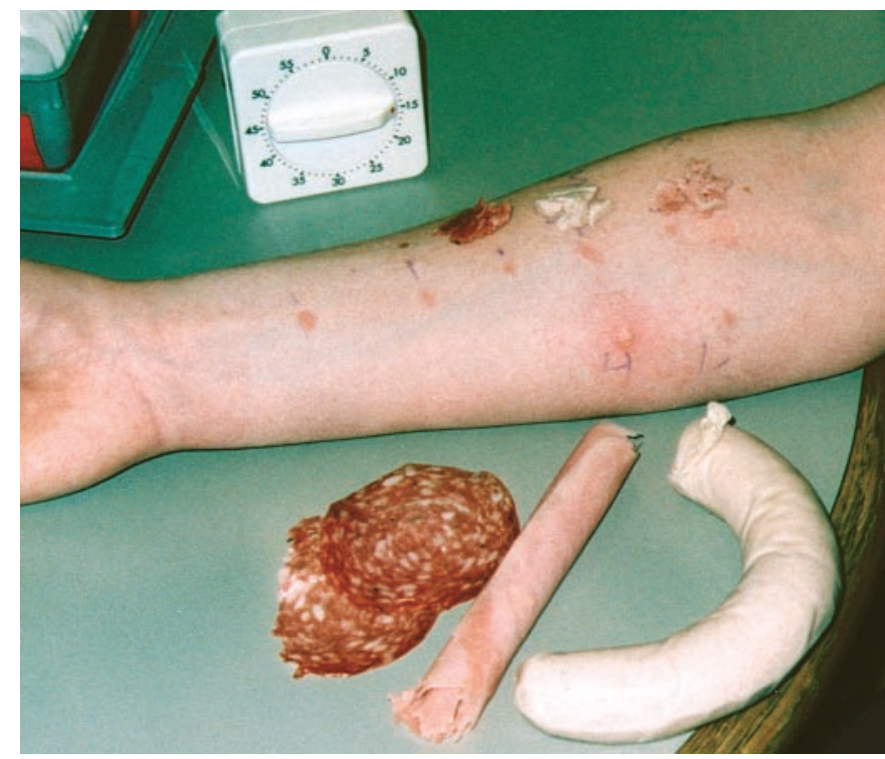

Abb. 2 Positive Prick-Prick-Teste auf Würste und Salami: Fleischallergien sind bei Erwachsenen auch unabhängig von einem „Chatporc“-Syndrom möglich (vgl. Fälle Nr. 6 und 7).

Dieser Fall entspricht den früher beschriebenen Kasuistiken einer Fleischallergie bei Erwachsenen mit typischer Latenz des Auftretens der Symptomatik nach Fleischgenuss [23].

\section{Fall 7}

Erstmals im Alter von 23 Jahren trat bei der Krankenschwester drei Stunden nach Genuss von „Chili con carne“ eine Urtikaria auf. Einen Monat darauf berichtete die Patientin über urtikarielle 
Effloreszenzen nach dem Verzehr von Rindfleisch und nochmals zwei Monate später über Urtikaria und Dyspnoe, welche sich zwei Stunden nach einem Mahl mit Lammfleisch einstellten. Bei der nachfolgenden notfallärztlichen Konsultation kollabierte die Patientin nach einer intravenösen Injektion von Steroiden. Entsprechend den Vermutungen der Patientin konnten sowohl in der Serologie (CAP Klasse 2) als auch in den Hauttestungen Sensibilisierungen gegen Fleischsorten wie Rind, Schwein und Lamm nachgewiesen werden. Das Atopie-Screening war unauffällig mit Ausnahme einer leichten Sensibilisierung gegen Katzenhaare im Intrakutan-Test (Prick-Test negativ) und einer deutlichen Erhöhung der spezifischen IgE gegen Katzenepithelien (CAP 3; 4,62 kU/l). Die Patientin, die keine Haustiere besitzt, verneinte respiratorische Symptome bei okkasioneller Katzenexposition.. Diese Konstellation könnte auf ein „Porc-chat-Syndrom“ hinweisen - primäre Sensibilisierung auf Schweinefleisch mit nachfolgender Kreuzreaktivität auf Katzenepithel -, in Analogie zum „Chat-porc-Syndrom“, bei welchem primär eine aktuelle Inhalationsallergie auf Katze mit nachfolgender Nahrungsmittelallergie auf Schweinefleisch berichtet wurde [24].

\section{Fall 8}

Die fast zehnjährige Patientin wurde allergologisch abgeklärt, weil häufig während der Turnstunden am Nachmittag ein urtikarielles Exanthem auftrat. Es wurde zunächst an eine Anstrengungsurtikaria gedacht, doch ein entsprechender Belastungstest war negativ und die Patientin konnte auch immer wieder Sport problemlos treiben. Die Hauttestungen mit Inhalationsallergenen und Nahrungsmitteln waren bis auf positive Prick-Test-Reaktionen auf Geflügelfleisch negativ und serologisch konnten beim negativem Sx1-spezifische IgE-Antikörper (CAP Klasse 3) gegen Poulet- und Putenfleisch nachgewiesen werden. Erst nachträglich gab die Patientin an, dass nach Genuss von Pouletfleisch, welches sie jeweils vor dem problematischen Turnunterricht verzehrte, ein Kratzen im Hals verspürte. Im Hinblick auf ein Vogel-Ei-Syndrom [25] wurden nachträglich noch IgE-Antikörper gegen Hühnerfedern, Hühnereigelb und Hühnereiweiß bestimmt, wobei die Befunde negativ waren. In Anbetracht der Anamnese und der Testergebnisse wurde die Diagnose einer so genannten „Food dependent exercise-induced“ Urtikaria bei monovalenter Sensibilisierung auf Pouletfleisch gestellt und eine strikte Geflügelfleisch-Eliminationsdiät verordnet. Anstrengungsabhängige Beschwerden sind in der Folge nicht mehr aufgetreten, ein Jahr nach Diagnosestellung ließ sich ein Rückgang der IgE-Sensibilisierung gegen Pouletfleisch von CAP-Klasse v 3 $(6,2 \mathrm{kU} / \mathrm{l})$ auf $2(1,87 \mathrm{kU} / \mathrm{l})$ feststellen.

Im Alter von etwa 13 Jahren manifestierte sich erstmals eine Rhinoconjunctivitis pollinosa. mit positiven Prick-Testen auf Gräserund Roggenpollen. Dazu gesellt sich im Alter von fünfzehn Jahren eine (latente) Sensibilisierung auf Birken- und Erlenpollen. Bezüglich der Geflügelfleisch-Allergie sind keine Episoden von akzidenziellen Einnahmen von Geflügeleiweißen mehr aufgetreten. Bei dieser jungen Patientin hat sich ihre Atopie offensichtlich zunächst als (monovalente) Nahrungsmittelallergie Typ C manifestiert und drei Jahre später ist sie an einer Rhinoconjunctivitis pollinosa erkrankt. Diese Tatsache lässt erahnen, dass sich in späteren Jahren eine Kombination der NMA Typ B entwickeln könnte.

\section{Fall 9}

Die Patientin beobachtete wiederholt Nasenlaufen und Engegefühl im Halsbereich nach Verzehr verschiedener eihaltiger Speisen. Sowohl in der Prick-Testung als auch serologisch fand sich eine Sensibilisierung gegenüber Eigelb (Prick +++, CAP Klasse 2), Eiklar (Prick +, CAP Klasse 2) und Hühnerfedern (Prick +, CAP Klasse 2; $1,76 \mathrm{kU} / \mathrm{l}$ ) bei sonst negativem Atopie-Screening. Vögel wurden zu Hause nie gehalten, entsprechende Symptome nach Kontakt mit federnhaltigem Bettzeug sind nie aufgetreten. Es handelt sich in diesem Falle wahrscheinlich um ein „Ei-VogelSyndrom“ [26,27] in Analogie zum „Vogel-Ei-Syndrom“ [25].

\section{Fall 10}

Dieser junge Patient mit Milchallergie und hochgradiger Kaseinsensibilisierung bemerkte erstmals im 14. Lebensjahr einen $\mathrm{Zu}-$ sammenhang zwischen der Einnahme von Kuhmilch und Auftreten von Atemnot. Die Nahrungsmittelanamnese in der Kindheit ist bland, es bestand jedoch ein frühkindliches perenniales Asthma bei schwacher Sensibilisierung gegen Hunde-, Katzenhaare und Rinderschuppen. Im weiteren Verlauf treten zusätzlich Urtikaria und rhinokonjunktivitische Symptome nach dem Genuss von Milchprodukten auf. Im Alter von 23 Jahren verlor der Patient während einem Status asthmaticus nach Verzehr von wenig Hüttenkäse das Bewusstsein, so dass eine Reanimation mit Intubation notwendig wurde. Serologisch wurde eine hochgradige Sensibilisierung gegenüber Kasein und entsprechend gegenüber Milcheiweißen festgestellt. Keine spezifischen IgE waren gegenüber Alpha-Lactalbumin und Beta-Lactoglobulin nachweisbar. Die Hauttestungen zeigten stark positive Sofortreaktionen gegenüber roher Kuhmilch und gegenüber Kasein bereits bei einer Verdünnung von 1:100 000 (Prick-Test). (Dieser Fall wurde in extenso andernorts publiziert) [28].

\section{Fall 11}

Die 27-jährige Patientin berichtete über vier Episoden einer allergischen Reaktion in Kombination mit Nahrungsaufnahme und physischer Anstrengung und/oder extremen Temperaturverhältnissen. Erstmals beklagte sie mit 22 Jahren eine Urtikaria nach einer Mahlzeit in Ägypten, zwei Jahre später erlitt sie eine schwere Urtikaria, Kreislaufkollaps mit Schwindel, Sehstörung und Amaurose nach einer Nudelmahlzeit und Exposition an der Kälte. Nach einer Brotmahlzeit traten ein Jahr später Urtikaria und Schwindel auf. Im Rahmen der letzten Episode, die sich im 32. Lebensjahr ereignete, bemerkte die Patientin auf der Skipiste 30 Minuten nach einer Mahlzeit (bestehend aus einem panierten Schnitzel mit Pommes frites und Ketchup) urtikarielle Quaddeln am Hals. Trotz der Einnahme eines Antihistaminikums (Terfenadine, insgesamt drei Tabletten) kam es zunächst zur generalisierten Urtikaria, Sehstörungen mit Dyspnoe und kurz darauf zu einem Kreislaufkollaps mit Bewusstlosigkeit und unkontrolliertem Stuhlabgang. Die Patientin musste aufgrund des anaphylaktischen Schocks notfallmäßig reanimiert und mit der Rega ins nächste Kantonsspital transportiert werden.

Im Rahmen der allergologischen Abklärung zeigte sich - bei einer Urticaria factitia - eine isolierte IgE-Sensibilisierung von CAP-Klasse 3 gegen Roggen- und Weizenmehl sowie gegen Gluten, bei negativen IgE-CAP-Ergebnissen auf Inhalationsallergene (Sx1 und Rx2). Bei der Bewertung der Anamnese und der Testergebnisse zeigte sich, dass die Patientin bei jeder dieser Mahlzei- 
ten Brotprodukte zu sich genommen hatte und sich zusätzlich entweder physisch anstrengte oder extremen Temperaturverhältnissen ausgesetzt war. Um die Konstellation einer so genannten „food dependent exercise-induced anaphylaxis“ [3] zu objektivieren, wurde auf der Allergiestation ein Provokationstest durchgeführt in Form eines Anstrengungstests mit und ohne vorheriger Brotaufnahme. Die Patientin entwickelte bei Anstrengung auf nüchternen Magen keine Symptome, es war auch kein Anstieg der Tryptase im Serum und eine Histaminausschüttung im Urin nachweisbar. Hingegen führte ein Anstrengungstest bei Zimmertemperatur nach Verzehr von Brot zu einer Urtikaria sowie zu einem leichten Anstieg des Histamins im Urin. Bei dem Krankheitsbild der „food dependent exercise-induced anaphylaxis" führt nur die Kombination von test-positiven Nahrungsmitteln, gefolgt von physischer Aktivität oder von physikalischen Reizen (wie z.B. Temperaturschwankungen) zu einer Mediatorenfreisetzung und dementsprechend zur klinischen Symptomatik einer allergischen Reaktion. Physische Anstrengung alleine und meistens auch die alleinige Nahrungsmittelaufnahme führen zu keiner Reaktion.

\section{Fall 12}

Erstmals in seinem Leben erlebte der 49-jährige Patient nach Genuss verschiedener Nüsse kurz nach einem Sauna-Besuch eine akute allergische Reaktion. Er berichtet über einen glühenden Kopf, Lippen- und Zungenschwellung, Dysarthrie, Atemnot sowie Kribbeln an den Handflächen. Die Symptome treten fünf Minuten nach Verzehr der Nüsse auf und sind nach 30 Minuten spontan regredient. Anhand der Hauttestungen Paranuss wird eine Nahrungsmittelallergie vom Typ C bei monovalenter Sensibilisierung gegen Paranüsse diagnostiziert.

\section{Fall 13}

Im Alter von 38 Jahren trat bei dieser Patientin vom Beruf Apothekerin und mit blander atopischer Vorbelastung, nach dem Verzehr einer Kiwifrucht eine schwere allergische Reaktion auf. Zunächst verspürte sie zehn Minuten nach dem Essens ein Kratzen im Hals begleitet von Urtikaria, nach weiteren 25 Minuten entwickelte sich ein Larynxödem und die Patientin kollabierte. Anamnestisch war ferner zu eruieren, dass sie seit einem Jahr keine Feigen mehr vertrug, nach deren Genuss traten nämlich Durchfall und Schweißausbrüche auf. Im täglichen Umgang mit Latex hatte die Apothekerin keine Probleme. Der Atopie-Screening war negativ, ebenfalls der CAP-Test mit Sx1 und Latex, der Prick-Test mit Kiwi-Extrakt und ein Prick-zu-Prick-Test mit frischer Feige hingegen waren stark positiv. Die Serologie war CAP Klasse 0 negativ für Kiwi, jedoch CAP Klasse 3 deutlich positiv für Feige. Im Prick-Test und serologisch (CAP Klasse 2, 1,26 kU/l) zeigte sich zusätzlich noch eine Sensibilisierung gegen Ficus benjamina, eine Zimmerpflanze, wovon eine in ihrem Wohnzimmer und eine zweite im Kinderzimmer stand. Eine häuslich-gebundene Respirationssymptomatik wurde nicht angegeben. Es scheint bei dieser nichtatopischen Patientin die Konstellation eines Feigen-Ficus-Syndroms $[29,30]$ ohne Latex-Sensibilisierung vorzuliegen, wobei die Feigen-Allergie wahrscheinlich primär per ingestionem, entsprechend dem Typ C erworben wurde, ebenfalls die Kiwi-Allergie. Da der CAP-Test auf Kiwi negativ war, ist die Kiwi-Sensibilisierung wahrscheinlich auch ingestiv, ohne Kreuzreaktivität mit Feige, entstanden.

\section{Fall 14}

Die Patientin ohne persönliche atopische Diathese berichtete über eine einmalige Episode im Alter von 28 Jahren: Drei Stunden nach einer Mahlzeit bestehend aus Lammfleisch, Kartoffeln und Salat (Kopfsalat, Karotten und Tomaten mit einer ansonsten problemlos tolerierten Fertig-Salatsauce) traten während eines Spaziergangs Husten, Abdominalkoliken, Urtikaria und Dyspnoe auf. Anhand erhöhter IgE-Antikörper (CAP Klasse 2) wurden Karotten und Kartoffeln als Allergie auslösende Nahrungsmittel identifiziert. Das Atopie-Screening mittels Hauttests war unauffällig, serologisch Sx1 CAP 2 ( = 1,26 kU/l) schwach positiv und auch rBetv 1 und rBetv 2 negativ. Weil die Patientin im Verlauf der Abklärungen schwanger wurde, konnten keine weiteren Testungen bezüglich Nahrungsmittelallergien vorgenommen werden.

\section{Fall 15}

Gemäß anamnestischen Angaben traten beim 34-jährigen Patienten nach einem Curry-Fischgericht aus der Tiefkühltruhe innerhalb von 30 Minuten ein Druckgefühl im Magen ohne Emesis, Erytheme, Pruritus und Atemnot auf. Fische konnten inzwischen problemlos toleriert werden. Bei blander persönlicher und familiärer Atopieanamnese waren beim Atopie-Screening positive Prick-Teste auf Gräser- und Roggenpollen sowie IgE-Antikörper (Sx1 CAP 3) nachweisbar. Von den Nahrungsmitteln zeigte sich eine monovalente Sensibilisierung gegen Curry bei der GewürzReihe auf. Obwohl der Patient die Durchführung von doppeltblinden, plazebo-kontrollierten oralen Provokationstesten mit Curry ablehnte, wurde eine Curry-Allergie als wahrscheinlich angenommen. Eine Lebensmittelvergiftung kam kaum infrage, da die Partnerin die gleiche Mahlzeit tolerierte.

\section{Fall 16}

Als Teenager absolvierte die nun 26-jährige Patientin eine Lehre in einem Fischrestaurant. Dabei traten erstmals in ihrem Leben beim Filetieren der Fische Schwellungen an den Fingern im Sinne einer Kontakt-Urtikaria auf. Etwa ein Jahr später bemerkte sie auch beim Verzehr von Fisch Beschwerden in Form eines Allergiesyndroms mit geschwollenen Lippen und Engegefühl im Hals. Nach Abschluss der Lehre traten zudem Pruritus und Schwellungen nach Kontakt mit diversen Salaten auf (Lollo rosso, weißer Chicorée, Kopfsalat und Endivien) (Abb. 3). Außerdem verspürte

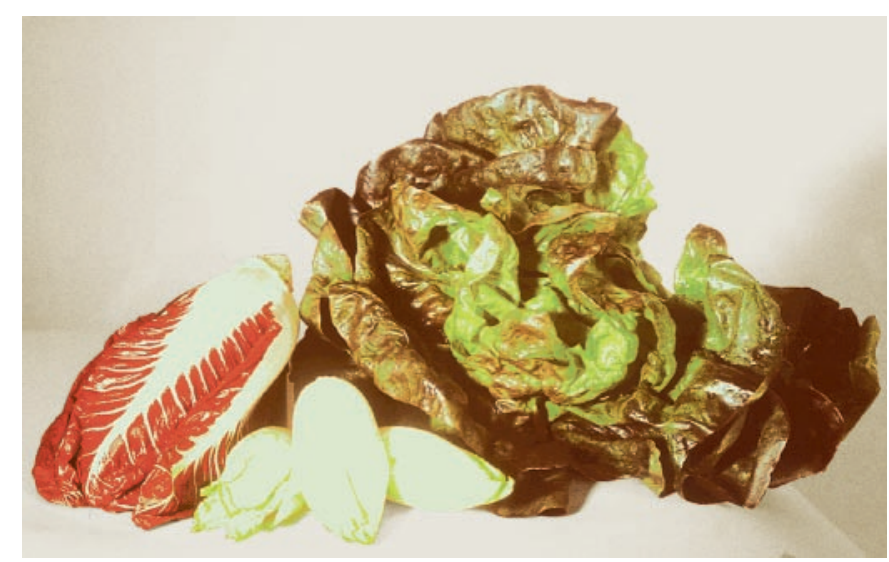

Abb. 3 Salate (roter Chicorée, Endivien [ = Brüsseler Salat]) und Kopfsalat gehören zu den Korbblütern und können ein Kontakt-UrtikariaSyndrom auslösen (vgl. Fall Nr. 16). 
sie nach der Einnahme von Kiwi und Tomaten ähnliche Symptome wie nach dem Verzehr von Fisch, nämlich Lippenschwellungen und Engegefühl im Hals. Im Alter von etwa 25 Jahren wurden zusätzlich konjunktivitische Beschwerden beim Zubereiten von Fisch ausgelöst. Sämtliche anamnestisch verdächtigten Allergie auslösenden Nahrungsmittel konnten mittels diverser Hautund Serologie-Teste bestätigt werden. Sowohl die familiäre wie auch die persönliche Anamnese und das Atopie-Screening waren bland bzw. negativ.

Obwohl die Nahrungsmittelallergie nicht im Kindesalter und ebenso wenig aufgrund von Kreuzreaktionen entstanden ist d.h. obwohl es sich um eine Nahrungsmittelallergie Typ C handelt - kann man nicht a priori ausschließen, dass die Sensibilisierung rein durch Hautkontakt im Sinne eines Kontakturtikariasyndroms [30] oder evtl. inhalativ erfolgt ist.

\section{Diskussion}

Bis anhin wurden Nahrungsmittelallergien bei Jugendlichen und Erwachsenen ohne manifeste atopische Vorerkrankung, entsprechend dem Nahrungsmittelallergie Typ C nach Pichler [4], als eher selten angenommen. Diesbezügliche Auswertungen über deren prozentuales Vorkommen unter einem Kollektiv von gesicherten Nahrungsmittelallergikern oder gar epidemiologischer Art, wurden jedoch bis anhin nicht durchgeführt. In einem Jahr konnten auf der Allergiestation der Dermatologischen Universitätsklinik Zürich unter 87 diagnostizierten Fällen von Nahrungsmittelallergien 16 Patienten vom Typ C ermittelt werden, was immerhin einem Anteil von $18,4 \%$ entspricht. In anderen Worten, etwa jeder fünfte Nahrungsmittelallergiker, welcher einen nicht-pädiatrischen Allergologen oder Dermatologen aufsucht, leidet über eine neue, im Laufe des Lebens erworbene Nahrungsmittelallergie, welche unter Umgehung der oralen Toleranz entstanden ist. Bei elf der 16 Fälle konnten als Allergene tierische Eiweiße (5-mal Krustentiere, 2-mal Fleisch, je 1-mal Fisch, Geflügel, Ei und Milch) ermittelt werden, bei fünf anderen waren Nahrungsmittelallergene pflanzlichen Ursprungs (je 1-mal Getreide, Paranuss, Kiwi, Feige, Karotte/Kartoffel und Curry) im Spiele. Die zwei Fälle von Fleischallergie bestätigen unsere früheren Beobachtungen, dass sich die Symptome nach Fleischgenuss nach einer Latenzzeit von einigen Stunden einstellen [23]. Als Ko-Faktoren wurde körperliche Anstrengung (3-mal) je einmal verbunden mit Kälteexposition oder Saunabesuch und einmal zusätzlich mit psychischem Stress und Rotweingenuss (multifaktorielle Auslösung). In einem Fall von Nahrungsmittelallergie nach Genuss großer Menge Flusskrebse war wahrscheinlich auch eine Histaminliberation im Spiele. Bezüglich des Sensibilisierungswegs entspricht der letzte Fall (Nr. 16) nicht ganz dem Typ C, da wahrscheinlich die Sensibilisierung zunächst perkutan erfolgte, im Sinne eines berufsbedingten Kontakturtikariasyndroms [31]. Nahrungsmittelallergie Typ C könnte somit, auch in Betracht der Möglichkeit einer beruflich erworbenen aerogenen Sensibilisierung bei einem Nichtatopiker, in drei Untergruppen weiter unterteilt werden: Typ C1 (ingestive Sensibilisierung), Typ C2 (aerogene Sensibilisierung durch Dämpfe, Stäube oder Aerosole) und Typ C3 (perkutane Sensibilisierung via Haarfollikel ohne präexistente atopische Dermatitis). Somit sollte in Zukunft auch bei einem Nichtatopiker mit akuter Symptomatik nach einer
Mahlzeit an eine Nahrungsmittelallergie gedacht werden und nach einer möglichen multifaktoriellen Auslösung gefragt werden.

\section{Literatur}

${ }^{1}$ Bruijnzeel-Koomen C, Ortolani C, Aas $\mathrm{K}$ et al. Adverse reactions to food. Position paper of the European Academy and Clinical Immunology. Allergy 1995; 50: 623-635

2 Wüthrich B, Etasamifar M. Nahrungsmittelallergien. Akt Dermatol 1995; 24: 369-376

${ }^{3}$ Wüthrich B. Klinik der Nahrungsmittelallergien. In: Jäger L, Wüthrich B (Hrsg). Nahrungsmittelallergien und -intoleranzen. Immunologie, Diagnostik, Therapie, Prophylaxe. München: Urban \& Fischer, 2002; 2. überarbeitete Auflage: $43-56$

${ }^{4}$ Pichler WJ. IgE-vermittelte Nahrungsmittelallergien. Klassifikation basierend auf dem Sensibilisierungsweg. Allergologie 1998; 9: $441-445$

${ }^{5}$ Crespo JF, Esteban M, Blanco C et al. Food allergy. A clinical and epidemiological study. J Allerg Clin Immunol (Abstract) 1992; 89: 192

${ }^{6}$ Bauer L, Ebner C, Hirschwehr R et al. IgE cross-reactivity between birch pollen, mugwort pollen and celery is due to at least three distinct cross-reacting allergens: immunoblot investigation of the birchmugwort-celery syndrome. Clin Exp Allergy 1996; 26: 1161 - 1170

${ }^{7}$ Bircher AJ, van Melle G, Haller E et al. IgE to food allergens are highly prevalent in patients allergic to pollens with and without symptoms of food allergy. Clin Exp Allergy 1994; 24: $367-374$

${ }^{8}$ Helbling A. Wichtige kreuzreaktive Allergene. Schweiz Med Wochenschr 1997; 127: $382-338$

${ }^{9}$ Stich O, Pichler WJ. Nahrungsmittelallergien bei Pollensensibilisierungen. Teil I: Kreuzreaktionen bei Birkenpollensensibilisierung. Allergologie 1993; 16: 288-294

${ }^{10}$ Pichler WJ, Stich O. Nahrungsmittelallergien bei Pollensensibilisierungen. Teil II: Kreuzreaktionen bei Beifußpollen-Sensibilisierungen. Allergologie 1993; 16: 494-501

${ }^{11}$ Wüthrich B, Hofer T. Nahrungsmittelallergie: das „Sellerie-Beifuß-Gewürz-Syndrom“. Assoziation mit einer Mangofrucht-Allergie? Dtsch Med Wochenschr 1984; 109: 981 - 986

12 Wüthrich B, Stäger J, Johansson SGO. Celery allergy associated with birch and mugwort pollinosis. Allergy 1990; 45: 566-571

${ }^{13}$ Etesamifar M, Wüthrich B. IgE-vermittelte Nahrungsmittelallergien bei 383 Patienten unter Berücksichtigung des oralen Allergie-Syndroms. Allergologie 1998; 21: $451-445$

${ }^{14}$ Mühlemann RJ, Wüthrich B. Nahrungsmittelallergien 1983-1987. Schweiz Med Wochenschrift 1991; 121: 1696 - 1700

15 Wüthrich B. Zur Nahrungsmittelallergie. Häufigkeit der Symptome und der allergieauslösenden Nahrungsmittel bei 402 Patienten - Kuhmilchallergie - Nahrungsmittel und Neurodermitis atopica. Allergologie 1993; 16: $280-287$

${ }^{16}$ Wüthrich B. Prävalenz. In: Jäger L, Wüthrich B (Hrsg). Nahrungsmittelallergien und -intoleranzen. Immunologie, Diagnostik, Therapie, Prophylaxe. München: Urban \& Fischer, 2002; 2. überarbeitete Auflage: $3-6$

17 Stöger P, Wüthrich B. Type I allergy to cow milk proteins in adults. A retrospective study on 34 adult milk-allergic patients. Int. Arch. Allergy Immunol 1993; 102: 399-407

18 Blötzer IC. IgE-vermittelte Nahrungsmittelallergien. Eine Auswertung bezüglich Klassifikation nach dem Sensibilisierungsweg anhand von 87 Fällen der Allergiestation der Dermatologischen Klinik des Universitätsspital Zürich des Jahres 1998. Zürich: Med. Diss., 2003

${ }^{19}$ Wüthrich B. Diagnostik. In: Jäger L, Wüthrich B. Nahrungsmittelallergien und -intoleranzen. Immunologie, Diagnostik, Therapie, Prophylaxe. München: Urban \& Fischer, 2002; 2. überarbeitete Auflage: 69-92

20 Daul CB, Slattery M, Reese G. Identification of the major brown shrimp (Penaeus aztecus) allergen as the muscle protein tropomyosin. Int Arch Allergy Appl Immunol 1994; 105: 49-55

${ }^{21}$ Hess-Schmid M, Wüthrich B. Zur Allergie auf Krevetten. Ein Beitrag über Reaktionen nach Genuss von Meeresfrüchten und Fischen. Hautarzt 1997; 48: $41-546$

22 Wittemann AM, Akkerdaas JH, van Leeuwen J et al. Identification of a cross-reactive allergen (presumably tropomyosin) in shrimp, mite and insects. Int Arch Allergy Appl Immunol 1994; 105: 56-61 
${ }^{23}$ Wüthrich B. Allergien auf Fleischeiweiße bei Erwachsenen. Allergologie 1996; 19: 130-134

24 Drouet M, Boutet S, Lauret M-G et al. Le syndrome porc-chat ou l'allergie croisée entre viande de porc et epithelia de chat. Allerg Immunol (Paris) 1994; 26: 166-172

${ }^{25}$ Mandallaz M, de Weck AL, Dahinden C. Bird-egg syndrome. Cross-reactivity between bird antigens and egg yolk livetins in IgE-mediated hypersensitivity. Int Arch Allergy Appl Immunol 1988; 87: 143 - 150

${ }^{26}$ Leser Chr, Hartmann AL, Praml G, Wüthrich B. The „Egg-Egg“ Syndrome: Occupational Respiratory Allergy to Airborne Egg Proteins with Consecutive Ingestive Egg Allergy in the Bakery and Confectionary Industry. J Invest Allergol Clin Immunol 2001; 11: 89-93

27 Wyss M, Huwyler T, Wüthrich B. „Bird-Egg“ and „Egg-Bird Syndrome“. Kreuzsensibilisierung zwischen inhalativen und ingestiven Vogelproteinen. Allergologie 1991; 14: 275-278

${ }^{28}$ Senti G, Leser CH, Wal J-M et al. Status asthmaticus und Anaphylaxie bei einem Erwachsenen mit hochgradiger Kuhmilchallergie. Schaf-, Ziegen- oder Stutenmilch als Alternative bei Milchproteinallergikern? Allergologie 2002; 25: $33-337$

29 Brehler R, Theissen U, Mohr C et al. „Latex-fruit syndrome“: frequency of cross-reacting IgE antibodies. Allergy 1994; 52: 404-410

${ }^{30}$ Dechamp C, Bessor J-C, Pauli G et al. First report of anaphylactic reaction after fig (Ficus carica) ingestion. Allergy 1995; 50: 514-516

31 Wüthrich B. Contact Urticaria and Protein Contact Dermatitis to Food. In: Marone G (ed). Clinical Immunology and Allergy in Medicine. Naples, Italy: S JGC Editions, 2003: 175-182

\section{Buchbesprechungen}

\author{
75 Jahre Hautklinik Berlin-Neukölln (127-2002) \\ P. K. Kohl (Hrsg) \\ Berlin: BMV, 2004. ISBN 3-88040-005-9
}

Die Klinik wurde 1927 gleichzeitig mit der Annahme des ersten Gesetzes zur Bekämpfung der Geschlechtskrankheiten eröffnet. Venerologie und Infektionskrankheiten waren und blieben ein Schwerpunkt. Beim Festakt anlässlich des 75-jährigen Bestehens konnte der jetzige Chefarzt Peter Kohl an den Gründungsdirektor Erich Langer (1927 - 1951, unterbrochen durch 12 Nazijahre) erinnern, dem zu Ehren eine Plakette in der Klinik angebracht ist, und seine beiden Vorgänger, Heinrich Teller (1951-1975) und Günter Ehlers (1975 - 1997) begrüßen. Die Venerologie hat die Klinik von den ursprünglich 80 Betten nach den Krieg vorübergehend auf 223 Betten anschwellen lassen. Eine Mikrosporie-Epidemie war Ausgangsargument für die Gründung der Kinderdermatologie, und der weit reichende Konsiliardienst mit Erste-Hilfe-Intervention stimulierte die Allergologie, wobei die Behandlung von Arzneimittelreaktionen besonders stark zugenommen hat.

Reinhard Breit aus München kam die Ehre der ersten Heinrich Teller Vorlesung zu, in welcher er die parallelen 75 Jahre der UVA Forschung nachzeichnete. Er begann mit der ersten biologischen Charakteristik durch K. W. Haußer und W. Vahle 1927 und führte bis in die weit gefächerte und differente therapeutische Nutzung. Ein gelungener, der Klinik würdiger Festakt und eine angemessene Publikation, fürwahr!

E. G. Jung, Heidelberg

\section{Aktuelle Trends in der pädiatrischen Dermatologie T. Jansen, D. Abeck, P. Altmeyer (Hrsg) \\ Berlin: BMV, 2004. 164 S., 65 Abb., 16 Tab. ISBN 3-88040-001-6}

Am 20. April 2002 fand in Bochum ein Symposium dieser Thematik statt, das weitgehend von Mitarbeitern der Dermatologischen Klinik der Ruhr-Universität Bochum bestritten wurde. Dies wird nun in einem Band von162 Seiten dargeboten. 6 interessante Kasuistiken sind vorangestellt und 15 Hauptbeiträge bieten Interessantes aus der Breite der Kinderdermatologie. Kurz sind sie gehalten, informieren und geben therapeutische Erfahrung weiter, die Beiträge, die recht uneinheitlich vorliegen. Auffrischung und Erweiterung ist die erklärte Absicht, die oft gut erreicht wird, gelegentlich aber auch vorwiegend der Wiedererkennung dienen kann. Post festum scheint der Elan der aktiven Autoren schon neuen Zielen zugewandt - verständlich. 